\title{
MXene Based Sensing Materials: Current Status and Future Perspectives
}

Vishnu Sankar Sivasankarapillai, ${ }^{1}$ Tata Sanjay Kanna Sharma, ${ }^{2,3}$ Kuo-Yuan Hwa ${ }^{2,3}$ Saikh Mohammad Wabaidur, ${ }^{4}$ Subramania Angaiah ${ }^{5}$ and Ragupathy Dhanusuraman ${ }^{1, *}$

\begin{abstract}
MXenes are a family of two-dimensional multifunctional materials in a rapid development phase since their discovery in 2011. MXenes possess excellent features like high conductivity and surface area, improved mechanical properties, hydrophilicity, and the ability to tune surface properties by modifying functional groups. These features make MXenes suitable candidates for a wide range of applications, including biomedical and energy storage. This review is focused on various types of MXenes which are recently been reported for sensor applications. The current scenario regarding the fabrication and properties of MXenes are initially described, followed by a discussion of their applications as piezoresistive and biochemical sensors. This involves mechanical strain detection and the detection of biomolecules, biomarkers, and drug molecules relevant to biomedical applications. Finally, the future perspectives are briefly discussed, which will help the researchers identify the limitations of the current scenario and develop new strategies that focus on developing novel, efficient and sensitive MXenebased sensors.
\end{abstract}

Keywords: MXene; Biosensors; Energy storage; Multifunctional materials; Biomedical applications.

Received: 12 October 2021; Accepted: 03 November 2021.

Article type: Review article.

\section{Introduction}

Two-dimensional materials have emerged as a platform of enormous possibilities in material science due to their distinctive electronic, mechanical, chemical, and optical properties. These materials are composed either of a single element such as Silicene, ${ }^{[1]}$ Graphene, ${ }^{[2]}$ Germanene, ${ }^{[3]}$ and Borophene ${ }^{[4]}$ or two elements such as dichalcogenides. ${ }^{[5]}$ MXenes are a relatively new candidate of this family, first

\footnotetext{
${ }^{1}$ Nano Electrochemistry Lab (NEL), Department of Chemistry,

National Institute of Technology Puducherry, Karaikal, 609-609, India.

${ }^{2}$ Department of Molecular Science and Engineering, National Taipei University of Technology, Taipei, Taiwan (R.O.C).

${ }^{3}$ Center for Biomedical Industry, National Taipei University of Technology, Taipei, Taiwan (R.O.C).

${ }^{4}$ Advanced Materials Research Chair, Chemistry Department, College of Science, King Saud University, Riyadh 11451, Saudi Arabia.

${ }^{5}$ Electro-Materials Research Laboratory, Centre for Nanoscience and Technology, Pondicherry University, Pondicherry 605 014, India.

*Email: ragu.nitpy@gmail.com;

ragu@nitpy.ac.in (R. Dhanusuraman)
}

discovered in 2011 by introducing titanium carbide $\left(\mathrm{Ti}_{3} \mathrm{C}_{2}\right)$. The synthesis method used $\mathrm{Ti}_{3} \mathrm{AlC}_{2}$ as the precursor in which the selective etching of $\mathrm{Al}$ atoms using hydrofluoric acid (HF) under ambient conditions giving $\mathrm{Ti}_{3} \mathrm{C}_{2}$ MXene as represented in Fig. 1. ${ }^{[6]}$

MXenes are derivatives of the three-dimensional MAX phases as the parent structure and have the general formula $M_{n+1} A X_{n}{ }^{[7]} M$ represents members from early transition metal families like scandium, titanium, vanadium, chromium, zirconium, niobium, molybdenum, hafnium, tantalum, and tungsten ( $\mathrm{Sc}, \mathrm{Ti}, \mathrm{V}, \mathrm{Cr}, \mathrm{Zr}, \mathrm{Nb}, \mathrm{Mo}, \mathrm{Hf}, \mathrm{Ta}, \mathrm{W}$, respectively), A indicates an element from either group 13 or group 14, and $\mathrm{X}$ can be either carbon or nitrogen. Here $\mathrm{n}$ is an integer ranging from 1 to 3 . MAX phase is a unique structure in which closely packed multilayers are present which are composed of alternating layers of $\mathrm{M}$ and $\mathrm{A}$. M-X bonds are strong compared to weak M-A bonds. MXenes are found to be superior to their parent MAX phases in terms of electronic, magnetic, optical, and electrochemical properties. The special feature of combined metallic conductivity of transition metal carbides/carbonitrides along with the presence of a hydrophilic terminated surface make MXenes a superior candidate among other two-dimensional materials. ${ }^{\left[{ }^{[8]}\right.}$ 


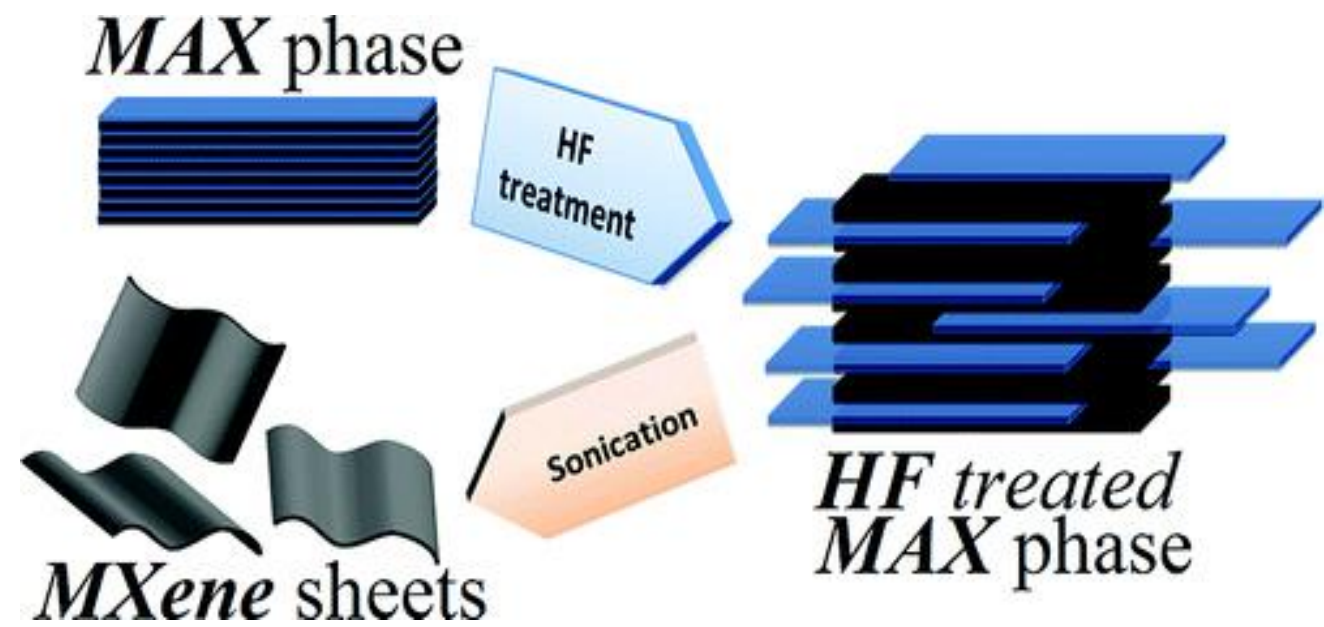

Fig. 1 Fabrication strategy of MXene by HF etching. Reproduced with permission from [6], Copyright 2012 American Chemical Society.

\subsection{Synthesis of MXenes}

Fabrication routes of MXenes are primarily focused on the removal of the more reactive " $A$ " atoms from $\mathrm{M}_{\mathrm{n}+1} \mathrm{X}_{\mathrm{n}}$ layers without any structural alterations. This feature is attributed to the relative weakness of M-A bonds compared to the M-X bonds, which is a stronger metallic covalent bonds. ${ }^{[8]}$ MAX phases are tightly wired to each other by strong van der Waals interaction, which complicates exfoliation more than similar 2D materials like Graphene, Phosphorene, etc. Literature suggests that around twenty MXenes are successfully produced to date from more than 70 types of MAX phases. The dissolving and etching of the A layers require aggressive acidic conditions, and hydrofluoric acid (HF) is conventionally employed. Fig. 2 represents the milestones in the history of the development of MXenes. Naguib et al. were the first group of researchers who successfully prepared MXene by etching the A layer from $\mathrm{Ti}_{3} \mathrm{AlC}_{2}$ using hydrofluoric acid (HF) ${ }^{[6]}$ Due to the hazardous nature of HF, researchers are focusing on developing novel alternative methods to prepare MXenes, giving the product in good yield. ${ }^{[9]}$ Here we discuss some feasible and effective methods for fabricating MXenes available in literature over the last decade.

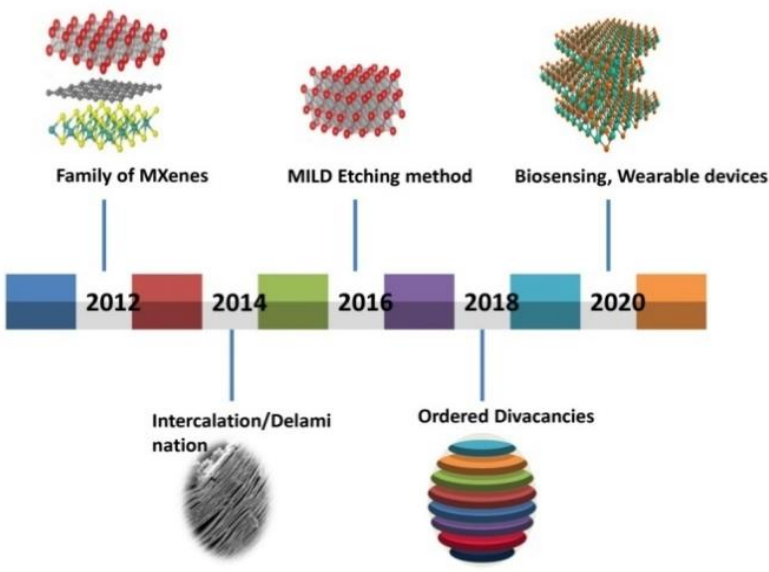

Fig. 2 Milestones in the development of MXenes.
Yang et al. developed an efficient etching technique based on the anodic corrosion of $\mathrm{Ti}_{3} \mathrm{AlC}_{2}$ without using fluorine in a binary aqueous electrolyte. The authors employed in situ intercalations of $\mathrm{NH}_{4} \mathrm{OH}$ after the dissolution of $\mathrm{Al}$, which resulted in the high yield, both mono- and bi-layers, extraction (over $90 \%$ ) of carbide flakes with up to $18.6 \mu \mathrm{m}$ size. ${ }^{[10]}$ Similarly, Sun et al. proposed a mechanism based on an intercalation-alloying expansion-microexplosion process for preparing layered fluoride-free $\mathrm{Ti}_{3} \mathrm{C}_{2} \mathrm{~T}_{\mathrm{x}}(\mathrm{T}=\mathrm{O}$ or $\mathrm{OH}){ }^{[11]}$ This rapid technique involved an etching strategy electrochemically without fluoride, leading to the Al layers being selectively corroded in titanium aluminum carbide $\left(\mathrm{Ti}_{3} \mathrm{AlC}_{2}\right)$.

Malaki et al. provide a detailed account of employing ultrasonication for preparing MXene as an effective and successful approach. ${ }^{[12]}$ The key process that needs to be considered is the proper dispersion and delamination of MXene flakes into layered sheets while producing MXenes since the properties of MXenes depend on the factors like layered structure, increased lateral size, and reduced defects, as well as the proper degree of exfoliation.

The two major difficulties encountered in the synthesis of nitride-based MXenes are higher formation energy of $M_{n+1} A N_{n}$ and compromised the stability of the $M_{n+1} N_{n}$ layers employed in the etchant (conventionally HF). Soundiraraju et $a l$. addressed this problem through the synthesis of Titanium Nitride $\left(\mathrm{Ti}_{2} \mathrm{~N}\right)$, where the $\mathrm{Al}$ atoms were selectively etched from ternary $\mathrm{Ti}_{2} \mathrm{AlN}{ }^{\left[{ }^{13]}\right]}$ The powder was immersed in a mixture of potassium fluoride and hydrochloric acid to achieve the intercalation of layers. Furthermore, the fewlayered $\mathrm{Ti}_{2} \mathrm{NT}_{\mathrm{x}}$ were obtained as a result of sonicating the formerly synthesized multilayered $\mathrm{Ti}_{2} \mathrm{NT}_{\mathrm{x}}$.

Aluminum fluoride $\left(\mathrm{AlF} \cdot 3 \mathrm{H}_{2} \mathrm{O}\right)$ is considered to be an undesirable byproduct most commonly obtained from the synthesis of MXenes. Cockreham et al. deduced the conditions leading to the formation of $\mathrm{AlF} \cdot 3 \mathrm{H}_{2} \mathrm{O}$ while employing $\mathrm{CoF}_{2} / \mathrm{CoF}_{3}$ for etching technique exploration. ${ }^{[14]}$ It 
is found that the ionic strength of the etching reagent plays a crucial role in the formation of $\mathrm{AlF} \cdot 3 \mathrm{H}_{2} \mathrm{O}$. The $\mathrm{AlF}_{3}$ complexation is stable with the ionic strength ranging between $\sim 8.5$ and $10 \mathrm{M}$. Alhabeb et al. synthesized $\mathrm{Ti}_{3} \mathrm{C}_{2}$ by selectively etching silicon from titanium silicon carbide $\mathrm{Ti}_{3} \mathrm{SiC}_{2}$, which is considered the most common MAX phase. ${ }^{[15]}$

A universal strategy was developed by Pang et al., which was based on an electrochemical etching route assisted thermally, for the synthesis of MXenes (e.g., $\mathrm{Ti}_{2} \mathrm{CT}_{\mathrm{x}}, \mathrm{Cr}_{2} \mathrm{CT}_{\mathrm{x}}$, and $\left.\mathrm{V}_{2} \mathrm{CT}_{\mathrm{x}}\right){ }^{\left[{ }^{16]}\right.}$ The experimental procedure is illustrated in Fig. 3. Since the strength of $\mathrm{M}-\mathrm{Al}$ and $\mathrm{M}-\mathrm{C}$ bonds are different, it was essential to determine the voltage for etching for individual MXene. The experiment was performed with the aid of cyclic voltammetry (CV) measurements. $\mathrm{Ti}_{2} \mathrm{AlC}$ has broad $\mathrm{CV}$ peaks, but unlike that, the plateaus corresponding to Al removal can also be identified. Tran et al. synthesized $\mathrm{V}_{4} \mathrm{C}_{3} \mathrm{~T}_{\mathrm{x}}$ based on the chemical exfoliation of the $\mathrm{V}_{4} \mathrm{AlC}_{3} \mathrm{MAX}$ phase by treatment with aqueous HF. ${ }^{[17]}$ Limbu et al. reported an ecofriendly and facile method for synthesizing reduced $\mathrm{Ti}_{3} \mathrm{C}_{2} \mathrm{~T}_{\mathrm{x}}$ MXene $\left(\mathrm{r}-\mathrm{Ti}_{3} \mathrm{C}_{2} \mathrm{~T}_{\mathrm{x}}\right)$ which involved treatment with $\mathrm{L}$ ascorbic acid at room temperature. ${ }^{[18]}$

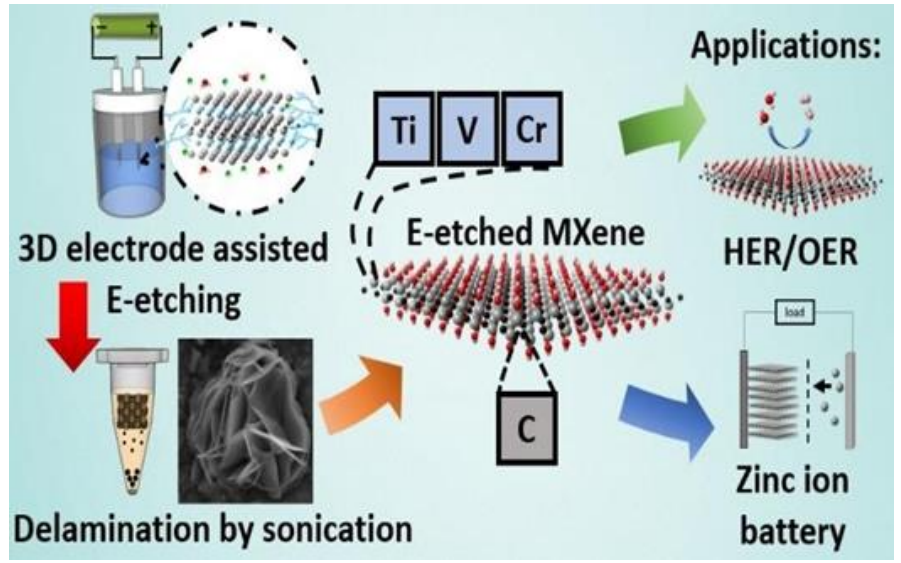

Fig. 3 Electrochemical etching route for preparing MXene for energy applications. Reproduced with permission from [16], Copyright 2019 American Chemical Society.

Urbankowski et al. demonstrated that $\mathrm{Mo}_{2} \mathrm{CT}_{\mathrm{x}}$ and $\mathrm{V}_{2} \mathrm{CT}_{\mathrm{x}}$ MXenes transform to two-dimensional (2D) metal nitrides $\mathrm{Mo}_{2} \mathrm{~N}$ and $\mathrm{V}_{2} \mathrm{~N}$ through ammoniation at $600{ }^{\circ} \mathrm{C} .{ }^{[19]}$ The $\mathrm{N}$ atoms that are obtained on the decomposition of ammonia molecules substitute the carbon atoms in the MXene precursor. Zhang et al. proposed two methodologies for synthesizing oxidized $\mathrm{Nb}_{2} \mathrm{CT}_{\mathrm{x}}$ MXene, the first being a hydrothermal method $\left(\mathrm{h}-\mathrm{Nb}_{2} \mathrm{O}_{5} / \mathrm{C}\right)$, and the second was the calcination of $\mathrm{CO}\left(\mathrm{c}-\mathrm{Nb}_{2} \mathrm{O}_{5} / \mathrm{C}\right) .{ }^{[20]}$ The authors revealed that the synergistic effect of the large lipophilic surface of the disordered carbon and the high dielectric constant of the $\mathrm{Nb}_{2} \mathrm{O}_{5}$ nanoparticles are the reason for superior electro-rheological performance. Zhao et al. reported a facile, single-step method for synthesizing nanosheets of Niobium carbide $\left(\mathrm{Nb}_{2} \mathrm{CT}_{\mathrm{x}}\right)$ MXene by a simplified approach in which $\mathrm{Nb}_{2} \mathrm{AlC}$ powder was etched. ${ }^{[21]}$

Frey et al. reported a positive and unlabeled (PU) Machine learning approach that can successfully predict which of the theoretically proposed MXenes have the highest probability of being synthesized successfully. ${ }^{[22]}$ The authors revealed 18 promising MXene candidates that can be possibly synthesized with the knowledge of elemental information and result from density functional theory (DFT) calculations of 2D transition metal carbides, carbonitrides, and nitrides, and their layered precursor MAX phases.

\subsection{MXene-based sensors}

The major applications of MXenes are represented in Fig. 4. The metal and the terminal surface groups of the MXene layer have a great deal of influence on the performance and the physical properties of the material.

It is noticed that the terminal surface groups influence the performance, and the optical and electronic properties of the material significantly, whereas the $\mathrm{M}$ in the MXene layer affects its electronic properties. The increasing research interest in the application of MXene for fabricating electrochemical sensors is due to the following features ${ }^{[8]}$ :

1. High conductivity

2. Good electroactive surface area

3. Ease of fabrication with other materials to give composite materials

4. Excellent hydrophilicity compared to other 2D materials

These properties allow fabricating sensors for various molecules, including bioanalytics, environmental contaminants, and other relevant chemical species like heavy metal ions and anionic analytes. In general, the electroanalytical performance of an analyte is often enhanced by the adsorption of the analyte at the electroactive surface. The terminal functional groups of the MXene and the $\mathrm{pH}$ of the analyte solution have been demonstrated to have a significant influence on the sensing action of MXene-based electrochemical sensors. For instance, MXenes have been reported for a good affinity for metal cations at higher $\mathrm{pH}$ due to the creation of a negative charge surface via ionization of functional groups. Thus, the intercalation and adsorption in the structural aspects of MXenes are effectively exploited in fabricating MXene-based electrodes for sensing applications. Zhu et al. performed the modification of $\mathrm{Ti}_{3} \mathrm{C}_{2} \mathrm{~T}_{\mathrm{x}}$ with $-\mathrm{OH}$ groups to provide an alkylated MXene which was further employed for the electrochemical detection of heavy metal ions such as $\mathrm{Cd}(\mathrm{II}), \mathrm{Pb}(\mathrm{II}), \mathrm{Cu}(\mathrm{II})$, and $\mathrm{Hg}(\mathrm{II})$, simultaneously, by using square wave voltammetry. ${ }^{[23]}$ In the experiment, the sensor developed showed high sensitivity with linear ranges between $0.1 \mu \mathrm{m}$ and $1.0 \mu \mathrm{m}$ for all the metals with a limit of detection values at $98 \mathrm{~nm}, 41 \mathrm{~nm}, 32 \mathrm{~nm}$, and $130 \mathrm{~nm}$, for $\mathrm{Cu}(\mathrm{II}), \mathrm{Pb}(\mathrm{II}), \mathrm{Cu}^{+}$, and $\mathrm{Hg}(\mathrm{II})$, respectively. Another sensor fabricated with $\mathrm{Ti}_{3} \mathrm{C}_{2} \mathrm{~T}_{\mathrm{x}}$ on a glassy carbon electrode was used to detect bromate $\left(\mathrm{BrO}^{3-}\right)$ ion where response between the linear range of $50 \mathrm{~nm}$ to $5 \mu \mathrm{m}$ was performed limit of detection (LOD) value of $41 \mathrm{nM}$ was observed. ${ }^{[24-26]}$

Hemoglobin $(\mathrm{Hb})$ and Tyrosinase are among the other enzymes reported to be used with MXenes to detect nitrite and phenol, respectively. Liu et al. developed a nitrite sensor by 


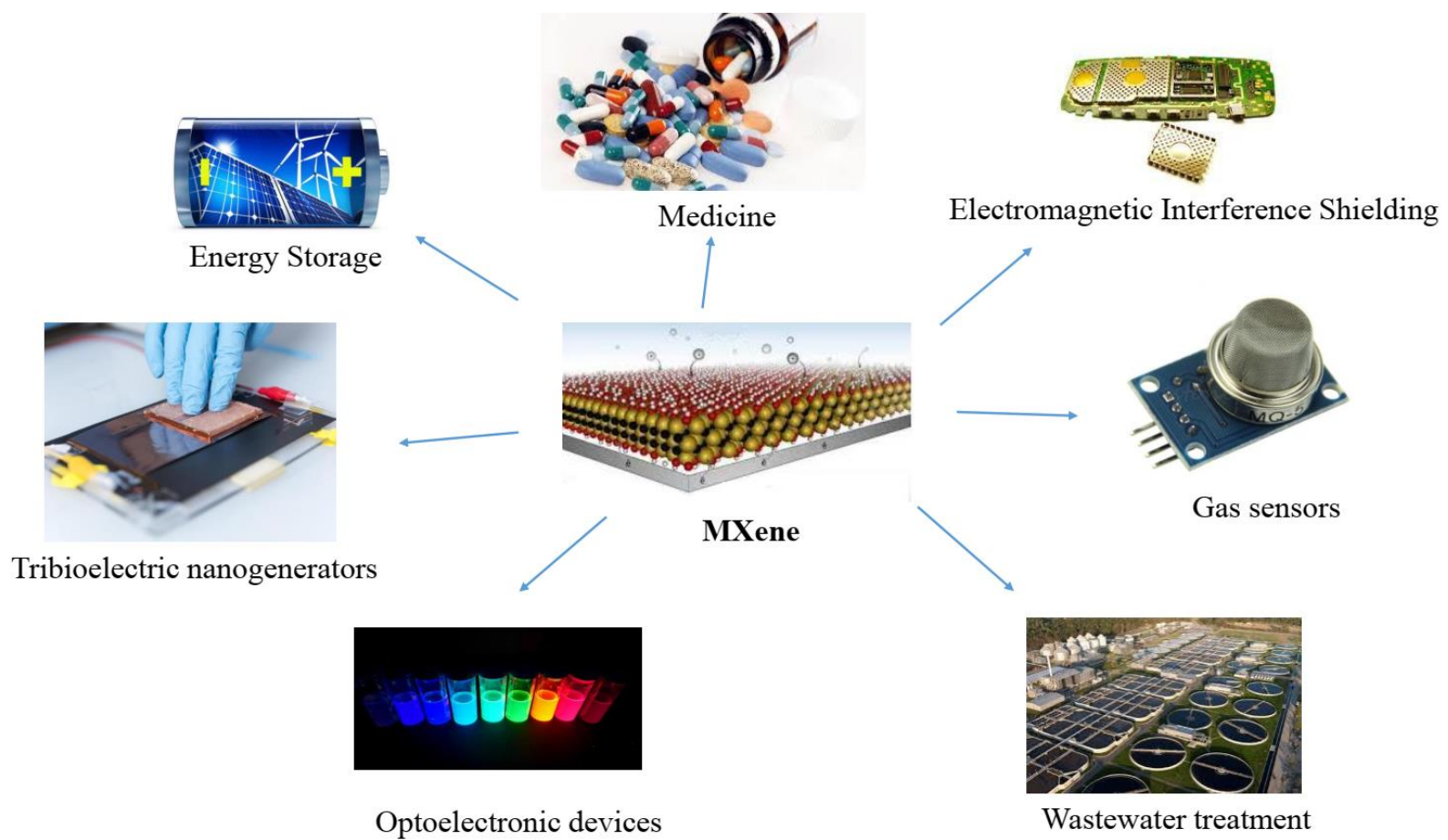

Fig. 4 Major applications of MXenes. Reproduced with permission from [7], Copyright 2020 Elsevier.

the immobilization of $\mathrm{Hb}$ on $\mathrm{Ti}_{3} \mathrm{C}_{2} \mathrm{~T}_{\mathrm{x}} \cdot{ }^{[27]} \mathrm{A}$ broad wave of reduction was observed at $-0.695 \mathrm{~V}$, which was attributed to the nitride being converted to $\mathrm{N}_{2} \mathrm{O}$. Here, a LOD of $0.12 \mu \mathrm{m}$ was achieved along with a broad linear range between $0.5 \mu \mathrm{m}$ to $11.8 \mathrm{~mm}$. Similarly, the alkylation of $\mathrm{Ti}_{3} \mathrm{C}_{2} \mathrm{~T}_{\mathrm{x}}$ was done to modify the surface of the MXene with $-\mathrm{OH}$ groups that underwent partial termination, thus providing an improved biocompatible microenvironment to immobilize Tyrosinase. ${ }^{[28]}$ This resulted in achieving a rapid time of response, coupled with an efficient electrocatalytic reaction, which was due to the rapid phenol diffusion onto the immobilized tyrosinase conjugated on the MXene nanoflakes, from the bulk solution. The sensor exhibited an excellent sensitivity of $414 \mathrm{~mA} \mathrm{~m}^{-1}$, with a very low LOD of $12 \mathrm{~nm}$.

Literature suggests that oxidation of $\mathrm{Ti}_{3} \mathrm{C}_{2} \mathrm{~T}_{\mathrm{x}}$ occurs at potentials higher than about $0.45 \mathrm{~V}$ vs $\mathrm{Ag} \mid \mathrm{AgCl}$ electrode, but this oxidation is not prominent when the metal nanoparticle is combined with $\mathrm{Ti}_{3} \mathrm{C}_{2} \mathrm{~T}_{\mathrm{x}}$ suggesting the stability attributed to metal nanoparticles. ${ }^{[29]}$ The mechanism of stability in the presence of metal nanoparticles is not much revealed but needs significant attention. This review briefly discusses two different aspects of MXene-based sensors such as piezoresistive (mechanical) sensors and electrochemical and luminescent biosensors. MXene-based mechanical sensors give a wide horizon of opportunities in the biomedical field like wearable electronics, ${ }^{[30]}$ therapeutic applications, ${ }^{[31]}$, etc. In contrast, biosensors cover sensors for detecting analytes such as biomolecules, biomarkers, and drug molecules. ${ }^{[32]}$

\section{Piezoresistive sensors}

Piezoresistive pressure sensors have got a significant interest in the biomedical field owing to their ability to monitor physiological signals. Wearable electronic sensors are the perfect examples of real-time applications of these sensors. ${ }^{[33]}$ Li et al. successfully developed a wearable electronic pressure sensor by incorporating multilayer $\mathrm{Ti}_{3} \mathrm{C}_{2} \mathrm{~T}_{x}$ MXene into poly(vinylidene fluoride) tetrafluoroethylene (PVDF-TrFE) which is a hydrophobic polymer. ${ }^{[34]}$ This piezoresistive pressure sensor works on the property of $\mathrm{Ti}_{3} \mathrm{C}_{2} \mathrm{~T}_{x}$ in which the resistance changes as a function of interlayer separation and is stable even after twenty-week exposure to air. Similarly, Liao et al. reported a similar wearable strain sensor prepared from conductive MXene nanocomposite organohydrogel $(\mathrm{MNOH}){ }^{[35]}$ It is prepared by the immersion of MXene nanocomposite hydrogel (MNH) into a solution of ethylene glycol (EG) and subsequent development into hydrogel polymer networks. This material possesses advantages over the typical conductive hydrogels which use pure water as the medium of dispersion and lose their properties due to freezing at subzero temperatures. The authors observed that $\mathrm{MNOH}$ could detect human physiological actions with a broad strain range (up to $350 \%$ strain) and possess outstanding features such as excellent anti-freezing properties $\left(-40^{\circ} \mathrm{C}\right)$, prolonged retention of moisture (8d), outstanding capability to self-heal, and enhanced mechanical properties.

Conducting polymers were also reported to be combined with MXenes for developing similar mechanical sensors. These polymers possess enhanced conductivity due to the conjugated carbon framework which facilitates charge transport. Due to this reason, conducting polymers have got a significant interest in energy storage devices and sensors. ${ }^{[36,37]}$ One example of this category of polymer is Polypyrrole which has already been well explored for its application in electrochemical sensors. ${ }^{[38]}$ Seroka et al. prepared 
functionalized $\mathrm{Ti}_{3} \mathrm{AlC}_{2}$-carbon nanoparticle-Polypyrrole nanocomposite having a sensitivity of $0.00017 \mathrm{kPa}^{-1}$ and a

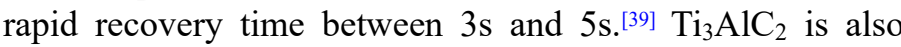
reported for fabricating $\mathrm{MXene}$ /ink sensors which have wide application prospects in monitoring the damage of structural composites used in aerospace applications. ${ }^{[40]}$ Proper investigation of employing conducting polymers for developing pressure sensors and correlating with their electrochemical performance can give rise to multifunctional sensors. $\mathrm{Ti}_{3} \mathrm{C}_{2} \mathrm{~T}_{\mathrm{x}} /$ polyurethane (PU) composite fibers were also reported as components for wearable strain-sensing textiles. ${ }^{[41]}$ Uzun et al. further extended the application potential of $\mathrm{Ti}_{3} \mathrm{C}_{2} \mathrm{~T}_{\mathrm{x}}$ by encapsulating cellulose yarns with $\mathrm{Ti}_{3} \mathrm{C}_{2} \mathrm{~T}_{\mathrm{x}}$ to form knittable and washable textile components. ${ }^{[42]}$ The electrospinning technique was also reported to develop 2D $\mathrm{Ti}_{3} \mathrm{C}_{2} \mathrm{~T}_{\mathrm{x}}$ MXene-based electrospun mats for piezoresistive sensing applications. ${ }^{[43]} \mathrm{Li}$ et al. fabricated a highly conductive MXene/cellulose nanocrystal (CNC) with super flexibility and coated it onto thermoplastic polyurethane (TPU) non-woven fabric (NWF). ${ }^{[4]}$ The authors used a dip-coating technique for the fabrication of wearable strain/pressure sensors. The material showed a wide range of sensing (about 83\%), an excellent sensitivity $(\mathrm{GF}=3405)$, and a very low limit of detection $(0.1 \%)$ which was primarily attributed to the opening or closing of micro-cracks present in the composite. A similar study reported the fabrication of a multilayer fiber sensor which was developed by self-assembling silver nanowire (AgNW), MXene, and waterborne polyurethane (WPU) layerby-layer assembly. ${ }^{[45]}$ This sensor proved to be highly sensitive and has a wide operating range (up to $100 \%$ ), along with exhibiting great stability (1000 cycles), reliability, a rapid response (344 ms), and relaxation time (344 ms). Similarly, Li et al. developed a sensor that exhibits ultrahigh sensitivity with a rapid time of response of $26 \mathrm{~ms}$, and excellent cycle stability (5600 cycles). ${ }^{[46]}$

Li et al. successfully developed a piezoresistive sensor by treating polyurethane (PU) with chitosan (CS) to obtain a positively charged CS@PU sponge. This sponge was successively coated with negatively charged $\mathrm{Ti}_{3} \mathrm{C}_{2} \mathrm{~T}_{\mathrm{x}}$ MXene sheets. ${ }^{[47]}$ The authors demonstrated that this sensor has a promising degree of compressibility and stable piezoresistive response for compressive strains of up to $85 \%$ with stress of $245.7 \mathrm{kPa}$.

Literature is available for employing aerogels for both electrochemical and piezoresistive sensor applications. ${ }^{[48]}$ Zhao et al. employed conductive $3 \mathrm{D}-\mathrm{Ti}_{3} \mathrm{C}_{2} \mathrm{~T}_{\mathrm{x}}$ porous architectures to develop hybrid aerogels by a combination of graphene oxide-assisted hydrothermal assembly and freezedrying. The structure of these hybrid aerogels consists of $\mathrm{Ti}_{3} \mathrm{C}_{2} \mathrm{~T}_{\mathrm{x}}$ walls in which the inner skeleton is graphene sheets, and this hybrid aerogel possesses future perspectives to be developed as a sensor material. ${ }^{[49]} \mathrm{Ma}$ et al. reported reduced graphene oxide ( $\mathrm{RGO}) / \mathrm{Ti}_{3} \mathrm{C}_{2} \mathrm{~T}_{\mathrm{x}}$ MXene aerogel with very good sensitivity $(22.56 \mathrm{kPa})$ and fast response time $(<200$ $\mathrm{ms}) .^{[50]}$ This study revealed that $\mathrm{RGO}$ nanosheets could prevent the poor oxidization of MXene by enhancing the wrapping of MXene inside the aerogel. Hydrogels were also reported for developing sensors with superior performance. ${ }^{[51]}$ Zhang et al. prepared MXenes by Yury mild method. ${ }^{[52]}$ Poly(N-iso-pracrylamide) (PNIPAM) hydrogel and physical crosslinking hydrogel were used as the matrix to prepare composite hydrogels' self-healing and stress-sensing properties. The conductivity of the composite hydrogel was about fifteen times more than that of the control hydrogel devoid of MXene.Tran et al. developed a thermal sensor using poly(2-dimethylamino) ethyl methacrylate) (PDMAEMA) which is a stimuli-responsive polymer with Ti-based MXene using the $\mathrm{OH}$ groups on the MXene surface as a linking agent for organic moieties. ${ }^{[53]}$ The authors also provide a theoretical insight into the conductivity mechanism of the material through DFT calculations. Yue et al. fabricated a piezoresistive sensor with an MXene-sponge network by a simple and efficient dip-coating process with insulating Polyvinyl alcohol (PVA) nanowires as a spacer. ${ }^{[54]}$ The authors achieved high sensitivity for the material for a broad pressure range with a low detection limit and excellent durability over 10,000 cycles. Zhang et al. give the possibility of developing wearable sensors based on $\mathrm{Ti}_{3} \mathrm{C}_{2}$ MXenes modified with small biomolecules to give enhanced conductivity and self-healing properties. ${ }^{[55]}$ This conductive composite consisting of $10 \mathrm{wt} \%$ of MXenes showed an elongation of $81 \%$ and a mechanical strength of $1.81 \mathrm{MPa}$.Yuan et al. used $\mathrm{Ti}_{3} \mathrm{C}_{2} \mathrm{~T}_{\mathrm{x}}$ with nylon fabric to form a sensor with gauge factors up to 24.35 and 5.98 within the strain range of $20 \%$ in the axial and radial of nylon fabric respectively. ${ }^{[56]}$ Also, the material possesses high durability and good fastness for washing which gives the possibility for developing real-life physiological sensors. Muckley et al. reported that films of $\mathrm{K}$ - and $\mathrm{Mg}$-intercalated MXenes exhibit relative humidity $(\mathrm{RH})$ detection thresholds of $\sim 0.8 \% \mathrm{RH}$ and showed monotonic $\mathrm{RH}$ response in the 0 $85 \% \mathrm{RH}$ range. ${ }^{[57]}$ This suggests a good potential for developing the material into an efficient humidity sensor.

Guo et al. present a biodegradable pressure sensor fabricated by sandwiching porous tissue paper incorporated with MXene with polylactic acid (PLA) thin sheets and PLA thin sheets. ${ }^{[58]}$ This flexible sensor was demonstrated to have high sensitivity with a low detection limit (10.2 Pa) with a fast response time.

It was demonstrated that the micro-architecture of the composites plays a significant role in the performance of piezoresistive sensors. This perspective has got attention in which the researchers are trying to design sensors with unique architecture. Shi et al. provide a good example of a biomimetic sensor that is based on "Brick-and-Mortar" architecture. ${ }^{[59]}$ Microscale hierarchical architecture is constructed from the combination of $\mathrm{Ti}_{3} \mathrm{C}_{2} \mathrm{~T}_{\mathrm{x}} / 1 \mathrm{D}$ silver nanowire "brick" and poly(dopamine)/Ni "mortar". The sensor worked efficiently using the loading energy to promote the stepwise propagation of cracks while stretching. Another biomimetic spider webinspired temperature and strain sensor are reported based on 
$\mathrm{Ti}_{3} \mathrm{C}_{2} \mathrm{~T}_{\mathrm{x}}$ hydrogels. Wang et al. developed this system based on 3D $\mathrm{Ti}_{3} \mathrm{C}_{2} \mathrm{~T}_{\mathrm{x}}$-polyacrylamide (PAM) nanocomposite hydrogels inspired by spider web architecture. ${ }^{[60]}$ The sensing properties of the material are attributed to the continuous distribution of MXene nanosheets connected by the PAM network which creates electron transport pathways and proton transport bridges in the hydrogel. Similarly, Urchin-like highly sensitive humidity sensor is also reported via the alkali oxidation method to grow in situ $\mathrm{TiO}_{2}$ nanowires on twodimensional $\mathrm{Ti}_{3} \mathrm{C}_{2}$ MXene which creates enhanced surface area. ${ }^{[61]}$ Chen et al. developed microspinous structures with a simple abrasive paper stencil printing process. ${ }^{[62]}$ The obtained sensor showed high sensitivity $(151.4 \mathrm{kPa})$, a short response time $(<130 \mathrm{~ms})$, and excellent cycle stability over 10,000 cycles. Another facile approach for the preparation of the sensor with a wrinkled structure is by spraying the active material onto the surface of a pre-stretched polyacrylate tape. ${ }^{[63]}$ Also, the sensor was demonstrated to have a sensitivity of $148.26 \mathrm{kPa}$ in a wide pressure range of up to $16 \mathrm{kPa}$ with excellent durability.

Song et al. fabricated hollow-structured MXenepolydimethylsiloxane composites (MPC) by utilizing nickel foam as the three-dimensional substrate for dip-coating. ${ }^{[64]}$ The resultant MPC performs a wide working range with bending angles of $0^{\circ}$ to $180^{\circ}$ and excellent long-term reliability up to 1000 cycles which can be employed as a bendable piezoresistive pressure sensor.

Electrospinning is one of the widely accepted fabrication methods of developing nanofibers with good performance for various applications like catalysis, ${ }^{[65]}$ drug delivery, ${ }^{[66]}$ energy storage, ${ }^{[67]}$ etc. Yang et al. developed MXene/polyurethane mat through electrospinning. ${ }^{[68]}$ The sensor showed high sensitivity, a low detection limit $(0.1 \%)$, and a large and tunable sensing range (up to $150 \%$ ).

Wang et al. reported the use of zero-dimensional silicon nanoparticles with MXene in cotton fabric to form a superhydrophobic textile sensor with a broad sensing range. ${ }^{[69]}$ This material exhibited superior sensitivity $\left(\mathrm{S}=12.23 \mathrm{kPa}^{-1}\right)$, a stable response under press-relaxing cycles, and broad sensing ranges (pressure: $8.8 \mathrm{~Pa}-70 \mathrm{kPa}$, bending: $0-180^{\circ}$, torsion: $\left.0-628 \mathrm{radm}^{-1}\right)$. Also, the conductivity is sustained under wet and corrosive conditions. Liu et al. observed that an increase in the amount of fluorine-containing functional groups also improved the hydrophobicity of the cotton fabric surface. ${ }^{[70]}$

Sharma et al. developed a highly sensitive sensor by sandwiching $\mathrm{Ti}_{3} \mathrm{C}_{2} \mathrm{~T}_{\mathrm{x}} / \mathrm{PVDF}-\mathrm{TrFE}$ composite nanofibrous scaffolds as a dielectric layer between biocompatible poly(3,4-ethylene dioxythiophene) polystyrene sulfonate /polydimethylsiloxane electrode. ${ }^{[71]}$ The authors demonstrated a high sensitivity of $0.51 \mathrm{kPa}$ with a detection limit of $1.5 \mathrm{~Pa}$ for the sensor along with high reliability of 10,000 cycles.

Recently Zheng et al. reported a novel method for the development of poly (3,4- ethylene dioxythiophene) (PEDOT) and $\mathrm{Ti}_{3} \mathrm{C}_{2} \mathrm{~T}_{\mathrm{x}}$ decorated cotton fabric strain sensors. ${ }^{[72]}$ The authors used vapor phase polymerization (VPP) and spraycoating strategy and achieved a low sheet resistance of 3.6 $\Omega \mathrm{sq}^{-1}$ and a high EMI shielding effectiveness of $36.62 \mathrm{~dB}$ at an applied voltage of $12 \mathrm{~V}$ along with good strain sensitivity. Metal nanoparticle-loaded MXenes are also reported as mechanical sensors. Li et al. reported such a work in which silver nanoparticles (AgNPs) were loaded on MXene nanosheets and compounded with one-dimensional silver nanowires (AgNWs) with high strain sensitivity. AgNPs built a bridge between AgNWs and MXene, ensuring continuity and a high gauge factor even at a large strain. ${ }^{[73]}$

\section{Electrochemical and Luminescence based sensors}

Biochemical sensors are always a 'hotspot' of electrochemical sensor research due to their vast application potential in the biomedical field. Highly sensitive and selective sensors which can detect biomolecules and drug molecules are always a significant area of research interest. Further electrochemical biosensors which can detect biomarkers with good performance in detecting diseases and metabolic disorders have also got good research attention over the last decade. Here we are discussing the most recent developments in biosensors which are fabricated using MXenes for application in the biomedical field.

One of the initial works reported for demonstrating the sensing action of MXene is the detection of Glucose. Rakhi et al. reported an interesting glucose biosensor based on $\mathrm{Au} / \mathrm{MXene}$ nanocomposites..$^{[74]}$ This study revealed that $\mathrm{Au}$ nanoparticles enhance the electron transfer process between the electrode and the Glucose oxidase enzyme. A linear response range for glucose detection was observed in the concentration range from 0.1 to $18 \mathrm{~mm}$ with a relatively high sensitivity of $4.2 \mu \mathrm{Amm}^{-1} \mathrm{~cm}^{-2}$ and a detection limit of $5.9 \mu \mathrm{m}$ with good reproducibility, repeatability, and sensitivity. Li et al. used a three-dimensional porous MXene/NiCo-LDH nanocomposite as a Glucose sensor. ${ }^{[75]}$ Good reproducibility and selectivity were obtained for the sensor with a working potential of $0.45 \mathrm{~V}$ (vs. SCE). The sensor also exhibited a broad linear range, a low limit of detection of $0.53 \mu \mathrm{M}$ and rapid response. It was observed that fast electron transfer rate and easy diffusion of electrolyte were attributed to porous $\mathrm{NiCo}-$ LDH nanosheets on the MXene substrate.

Kadirsoy et al. successfully developed a quartz crystal microbalance (QCM) sensor for the detection of chlorpyrifos, a common broad-spectrum organophosphate insecticide using delaminated sulfur-doped MXene (d-S- $\mathrm{Ti}_{3} \mathrm{C}_{2} \mathrm{Tx}$ MXene) ${ }^{[76]}$ The sensor possesses a linear response range of $1.0 \times 10^{-12}$ $1.0 \times 10^{-10} \mathrm{M}$ with a detection limit of $3.0 \times 10^{-13} \mathrm{M}$.

It is evident that the aggregation of MXene flakes significantly affects their electrocatalytic activity. Thus, delamination approaches like sonication are reported, which can prevent the aggregation of MXene flakes. Ma et al. developed a sensor for the detection of Fisetin using the selfassembly of negatively charged $\mathrm{Ti}_{3} \mathrm{C}_{2} \mathrm{~T}_{\mathrm{x}}$ MXene flakes and positively charged amine-functionalized carbon nanotubes 
$\left(\mathrm{NH}_{2}-\mathrm{CNTs}\right){ }^{[83]}$ Fisetin is a dietary antioxidant used for various ailments in humans including the enhancement of chemotherapeutic drugs. ${ }^{[84]}$ This study revealed the ability of $\mathrm{NH}_{2}$-CNTs to form well-defined porous structures by preventing the aggregation of MXene flakes and thereby significantly enhancing the electrocatalytic activity. The sensor was successfully used in real samples and showed a linear detection range for Fisetin concentration range 0.003 $\mu \mathrm{molL}{ }^{-1}$ to $20.0 \mu \mathrm{molL}^{-1}$ with a detection limit of $1.0 \mathrm{nmolL}^{-1}$.

Ammonia sensors always have a significant area of interest owing to their adverse effects on the environment. Wu et al. provided a good example of employing MXene-based sensors to monitor environmental pollutants like ammonia at room temperature. ${ }^{[77]}$ The authors prepared $\mathrm{Ti}_{3} \mathrm{C}_{2}$ MXene by etching off $\mathrm{Al}$ atoms from $\mathrm{Ti}_{3} \mathrm{AlC}_{2}$ and coating it on the surface of ceramic tubes to construct gas sensors. It was observed that the material has good selectivity for $\mathrm{NH}_{3}$ with a linear response in the concentration range from 10 to $700 \mathrm{ppm}$ at room temperature. Xiao et al. used first principle simulation studies and observed that $\mathrm{NH}_{3}$ could be strongly adsorbed on O-terminated semiconducting $\mathrm{MXenes} \mathrm{M}_{2} \mathrm{CO}_{3}(\mathrm{M}=\mathrm{Sc}, \mathrm{Ti}, \mathrm{Zr}$, and $\mathrm{Hf}$ ), which renders them the potential candidates as the $\mathrm{NH}_{3}$ sensor. ${ }^{\left[{ }^{[8]}\right.}$ Further, the authors observed that $\mathrm{Zr}_{2} \mathrm{CO}_{3}$ as an example, is highly selective towards $\mathrm{NH}_{3}$ against other common gas molecules, and the adsorption energy dramatically increases from $-0.81 \mathrm{eV}$ to $-0.20 \mathrm{eV}$ when extra two electrons are injected into the $\mathrm{Zr}_{2} \mathrm{CO}_{3}$ sheet of $3 \times 3$ dimensions.

Chen et al. employed the excitation-dependent blue photoluminescence (PL) of $\mathrm{Ti}_{3} \mathrm{C}_{2}$ quantum dots for fabricating intracellular $\mathrm{pH}$ sensors by combining with $\left[\mathrm{Ru}(\mathrm{dpp})_{3}\right] \mathrm{Cl}_{2}$. The authors used a sonication cutting and hydrothermal approach for the surface modification of $\mathrm{Ti}_{3} \mathrm{C}_{2}$ and demonstrated a high $\mathrm{PL}$ response attributed to the deprotonation of surface defects. ${ }^{[79]}$

Yuan et al. developed a three-dimensional (3D) MXene framework through combined electrospinning and selfassembly approach for the detection of volatile organic compounds. ${ }^{[80]}$ The sensor exhibited a wide detection range from $50 \mathrm{ppb}$, fast response, and recovery time ( $<2 \mathrm{~min})$, with retention of performance for 1000 bending cycles for various species such as acetone, methanol, and ethanol.

Fang et al. used negatively charged $\mathrm{OH}$-functionalized composite film with Nafion for the fabrication of a solid-state electro-chemiluminescent sensor. ${ }^{[81]}$ The authors reported long-term stability and a very low detection limit for the sensing of Tripropylamine. Further, the real-sample analysis of the sensor was demonstrated using single-nucleotide mismatch discrimination in human urine, which got promising results.

Sharma et al. reported that a $\mathrm{Ti}_{3} \mathrm{C}_{2} \mathrm{O}_{2}$-based probe can provide superior sensing of Cortisol with a detection limit of $15.7 \mathrm{fg} / \mathrm{mL} .^{\left[{ }^{[82}\right]}$ This MXene-based Cortisol sensor is relevant for real-time applications since it provides a non-invasive fiber optic surface plasmon resonance (FOSPR) based sensor probe to detect salivary Cortisol at the wavelength of $830 \mathrm{~nm}$.

Apart from Titanium-based MXenes, other MXenes are also in their initial stage of exploration. One such study was reported by Lee et al., which employed the use of vanadium carbide MXene $\left(\mathrm{V}_{2} \mathrm{CT}_{\mathrm{x}}\right)$ for non-polar gas sensing applications. ${ }^{[85]}$ The sensor consists of single-/few-layer twodimensional $\mathrm{V}_{2} \mathrm{CT}_{\mathrm{x}}$ on polyimide film and showed ultrahigh sensitivity at room temperature.

\section{Scope and future perspectives}

MXenes are in a significant pace of development and got exciting research interest in various applications like hydrogen storage, catalysis, supercapacitors, batteries, desalination, etc. Apart from this, the fabrication of MXene-based sensors paved the way for the horizon of opportunities in the detection of different categories of target molecules. Some of the most relevant candidates for these target molecules include environmental pollutants, biomolecules, drug molecules, and other harmful chemical entities. Further, the categories of piezoresistive MXene sensors are also a developing area that possesses exciting opportunities for the design and development of wearable electronics. But the area is still in a budding phase which needs enough research attention to make sensors that can be applied to a wide range of applications and to detect novel and relevant analytes. We would like to invite the attention of readers to the following aspects of MXene research to accelerate the journey which gives new opportunities for MXene-based sensor devices as illustrated in Fig. 5.

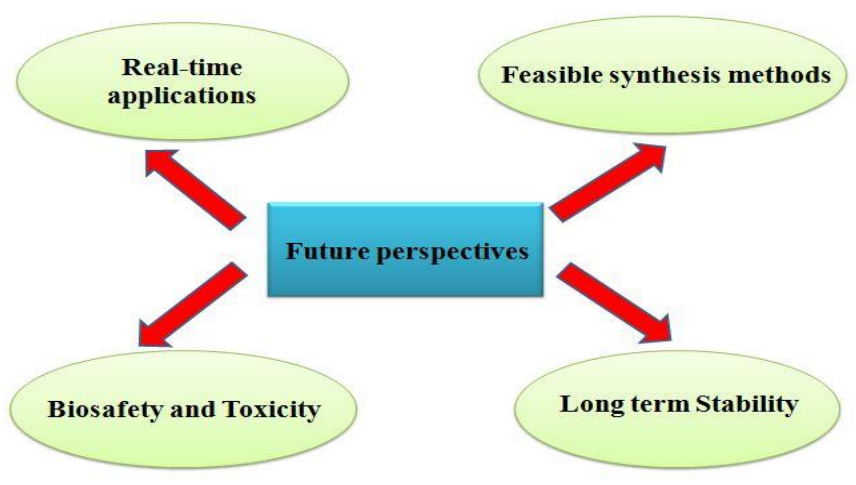

Fig. 5 Future directions of MXene research.

- The necessity for novel and cost-effective synthesis strategies: Even though more studies are emerging which demonstrate modified strategies for the preparation of MXenes, the design and development of MXenes are not much shifted from conventional chemical strategies. This makes the fabrication method tedious which affects the options for getting appropriately designed MXenes for specific applications. Microwaves and Ultrasound assisted methods should be considered to improve the preparation process. 
- The gap between theory and practice: There are many MXenes that are reported for their stable existence in literature through theoretical predictions. These approaches involve high throughput screening and density functional calculations which gives the idea of elemental combinations that produce stable MXenes. But most of the studies are still focused on Titanium based MXenes like $\mathrm{Ti}_{3} \mathrm{C}_{2} \mathrm{~T}_{x}$, which are produced by experimental preparation methods. Urgent attention is needed in this perspective which could give rise to novel formulations of MXene, which probably possess superior properties.

- Lack of long-term stability for sensor applications: It has been observed that both single and multilayered MXenes like $\mathrm{Ti}_{3} \mathrm{C}_{2} \mathrm{~T}_{\mathrm{x}}$ will degrade with humidity and an aqueous environment. This problem limits the application of MXenes for fabricating into stable sensor probes which need to be operated in hydrophilic conditions. Thus, other types of MXenes have been synthesized which is having good conductivity along with high stability to be used as sensors. Weak gelation of MXene layers is another performance for different applications. the major problem that significantly affects their morphology

- Biosafety and toxicity: Even though some studies analyzed the toxicity aspects of MXenes, this perspective is still not explored in detail. Toxicity, including both cytotoxicity and genotoxicity, along with the biosafety aspect has to be thoroughly investigated before their realtime application as sensors.

\section{Conclusion}

MXenes have amazing research interest within a short period after their discovery due to their exciting layered structure and metallic-like conductivity. Researchers across the world working on sensors are shifting their focus to novel twodimensional materials like MXenes which can show superior performance over conventional materials. The good conductivity and high stability of MXenes make them an ideal candidate for the fabrication of sensors that can be employed for diagnostic applications. On the other hand, this area still needs urgent attention for making a new category of MXenes other than Titanium-based materials. Even though theoretical studies are increasing the choice of preparing MXene-based sensors from other transition metals, most of the works in this area are not successfully explored other varieties. There is a high probability and scope of developing a new category of MXene candidates which might possess good performance over the reported materials. We hope this review will help the researchers understand the current scenario existing in MXene based sensors that have not attained good scientific attention and high possibility of novel discoveries.

\section{Acknowledgments}

The authors would like to thank for the basic research support from National Institute of Technology Puducherry, Karaikal, India.

\section{Conflict of Interest}

There is no conflict of interest.

\section{Supporting Information}

Not Applicable.

\section{References}

[1] M. Houssa, A. Dimoulas, A. Molle, Journal of Physics: Condensed Matter, 2015, 27, 253002, doi: 10.1088/09538984/27/25/253002.

[2] Y. Shao, J. Wang, H. Wu, J. Liu, I. Aksay, Y. Lin, Electroanalysis, 2010, 22, 1027-1036, doi: 10.1002/elan.200900571.

[3] A. Acun, L. Zhang, P. Bampoulis, M. Farmanbar, A. van Houselt, A. N. Rudenko, M. Lingenfelder, G. Brocks, B. Poelsema, M. I. Katsnelson, H. W. Zandvliet, Journal of Physics: Condensed Matter, 2015, 27, 443002, doi: 10.1088/09538984/27/44/443002.

[4] J. Joseph, V. S. Sivasankarapillai, S. Nikazar, M. S. Shanawaz, A. Rahdar, H. Lin, G. Z. Kyzas, ChemSusChem, 2020, 13, 37543765, doi: $10.1002 /$ cssc. 202000782 .

[5] Y.-H. Wang, K.-J. Huang, X. Wu, Biosensors and Bioelectronics, 2017, 97, 305-316, doi: 10.1016/j.bios.2017.06.011.

[6] M. Naguib, O. Mashtalir, J. Carle, V. Presser, J. Lu, L. Hultman, Y. Gogotsi, M. W. Barsoum, ACS Nano, 2012, 6, 13221331, doi: 10.1021/nn204153h.

[7] V. S. Sivasankarapillai, A. K. Somakumar, J. Joseph, S. Nikazar, A. Rahdar, G. Z. Kyzas, Nano-Structures \& NanoObjects, 2020, 22, 100457, doi: 10.1016/j.nanoso.2020.100457.

[8] T Yu, C B Breslin, Journal of The Electrochemical Society, 2019, 167, 037514, doi: 10.1149/2.0142003JES.

[9] X. Li, M. Li, Q. Yang, G. Liang, Z. Huang, L. Ma, D. Wang, F. Mo, B. Dong, Q. Huang, C. Zhi, Advanced Energy Materials, 2020, 10, 2001791, doi: 10.1002/aenm.202001791.

[10] S. Shylesh, A. Wagener, A. Seifert, S. Ernst, W. Thiel, Angewandte Chemie International Edition, 2010, 49, 184-187, doi: 10.1002/anie.200903985.

[11] Z. Sun, M. Yuan, L. Lin, H. Yang, C. Nan, H. Li, G. Sun, X. Yang, ACS Materials Letters, 2019, 1, 628-632, doi: 10.1021/acsmaterialslett.9b00390.

[12] M. Malaki, A. Maleki, R. S. Varma, Journal of Materials Chemistry A, 2019, 7, 10843-10857, doi: 10.1039/c9ta01850f.

[13] B. Soundiraraju, B. K. George, ACS Nano, 2017, 11, 88928900, doi: 10.1021/acsnano.7b03129.

[14] C. B. Cockreham, X. Zhang, H. Li, E. Hammond-Pereira, J. Sun, S. R. Saunders, Y. Wang, H. Xu, D. Wu, ACS Applied Energy Materials, 2019, 2, 8145-8152, doi: 10.1021/acsaem.9b01618.

[15] S. H. Yang, K. L. Wu, Y. Chi, Y. M. Cheng, P. T. Chou, Angewandte Chemie, 2011, 123, 8420-8424, doi: 10.1002/ange.201103515.

[16] S. Y. Pang, Y. T. Wong, S. Yuan, Y. Liu, M. K. Tsang, Z. Yang, H. Huang, W. T. Wong, J. Hao, Journal of the American Chemical Society, 2019, 141, 9610-9616, doi: 10.1021/jacs.9b02578. 
[17] M. H. Tran, T. Schäfer, A. Shahraei, M. Dürrschnabel, L. Molina-Luna, U. I. Kramm, C. S. Birkel, ACS Applied Energy Materials, 2018, 1, 3908-3914, doi: 10.1021/acsaem.8b00652.

[18] T. B. Limbu, B. Chitara, J. D. Orlando, M. Y. Garcia Cervantes, S. Kumari, Q. Li, Y. Tang, F. Yan, Journal of Materials Chemistry C, 2020, 8, 4722-4731, doi: 10.1039/c9tc06984d.

[19] P. Urbankowski, B. Anasori, K. Hantanasirisakul, L. Yang, L. Zhang, B. Haines, S. J. May, S. J. L. Billinge, Y. Gogotsi, Nanoscale, 2017, 9, 17722-17730, doi: 10.1039/c7nr06721f.

[20] W. L. Zhang, J. Tian, H. Zeng, J. Liu, Y. Tian, Chemical Engineering Journal, 2019, 366, 321-329, doi: 10.1016/j.cej.2019.02.065.

[21] J. Zhao, J. Wen, L. Bai, J. Xiao, R. Zheng, X. Shan, L. Li, H. Gao, X. Zhang, Dalton Transactions, 2019, 48, 14433-14439, doi: $10.1039 / \mathrm{c} 9 \mathrm{dt} 03260 \mathrm{f}$.

[22] N. C. Frey, J. Wang, G. I. Vega Bellido, B. Anasori, Y. Gogotsi, V. B. Shenoy, ACS Nano, 2019, 13, 3031-3041, doi: 10.1021/acsnano.8b08014.

[23] J. Zhu, E. Ha, G. Zhao, Y. Zhou, D. Huang, G. Yue, L. Hu, N. Sun, Y. Wang, L.Y. S. Lee, C. Xu, K.Wong, D. Astruc, P. Zhao, Coordination Chemistry Reviews, 2017, 352, 306-327, doi: 10.1016/j.ccr.2017.09.012.

[24] P. A. Rasheed, R. P. Pandey, K. Rasool, K. A. Mahmoud, Sensors and Actuators B: Chemical, 2018, 265, 652-659, doi: 10.1016/j.snb.2018.03.103.

[25] L. Zhou, X. Zhang, L. Ma, J. Gao, Y. Jiang, Biochemical Engineering Journal, 2017, 128, 243-249, doi: 10.1016/j.bej.2017.10.008.

[26] D. Song, X. Jiang, Y. Li, X. Lu, S. Luan, Y. Wang, Y. Li, F. Gao, Journal of Hazardous Materials, 2019, 373, 367-376, doi: 10.1016/j.jhazmat.2019.03.083.

[27] H. Liu, C. Duan, C. Yang, W. Shen, F. Wang, Z. Zhu, Sensors and Actuators B: Chemical, 2015, 218, 60-66, doi: 10.1016/j.snb.2015.04.090.

[28] L. Wu, X. Lu, Dhanjai, Z.-S. Wu, Y. Dong, X. Wang, S. Zheng, J. Chen, Biosensors and Bioelectronics, 2018, 107, 69-75, doi: 10.1016/j.bios.2018.02.021.

[29] L. Lorencova, T. Bertok, J. Filip, M. Jerigova, D. Velic, P. Kasak, K. A. Mahmoud, J. Tkac, Sensors and Actuators B: Chemical, 2018, 263, 360-368, doi: 10.1016/j.snb.2018.02.124.

[30] Y. Zhang, M. Gong, P. Wan, Matter, 2021, 4, 2655-2658, doi: 10.1016/j.matt.2021.06.041.

[31] S. P. Sreenilayam, I. Ul Ahad, V. Nicolosi, D. Brabazon, Materials Today, 2021, 43, 99-131, doi: 10.1016/j.mattod.2020.10.025.

[32] R. Khan, S. Andreescu, Sensors, 2020, 20, 5434, doi: $10.3390 / \mathrm{s} 20185434$.

[33] L. Rum, O. Sten, E. Vendrame, V. Belluscio, V. Camomilla, G. Vannozzi, L. Truppa, M. Notarantonio, T. Sciarra, A. Lazich, A. Mannini, E. Bergamini, Sensors, 2021, 21, 1858, doi: $10.3390 / \mathrm{s} 21051858$.

[34] L. Li, X. Fu, S. Chen, S. Uzun, A. S. Levitt, C. E. Shuck, W. Han, Y. Gogotsi, ACS Applied Materials \& Interfaces, 2020, 12, 15362-15369, doi: 10.1021/acsami.0c00255.
[35] H. Liao, X. Guo, P. Wan, G. Yu, Advanced Functional Materials, 2019, 29, 1904507, doi: 10.1002/adfm.201904507.

[36] Z. Gan, N. Song, H. Zhang, Z. Ma, Y. Wang, C. Chen, Journal of the Electrochemical Society, 2020, 167, 085501, doi: 10.1149/1945-7111/ab8c82.

[37] Y. Liu, N. Song, Z. Ma, K. Zhou, Z. Gan, Y. Gao, S. Tang, C. Chen, Materials Chemistry and Physics, 2019, 223, 548-556, doi: 10.1016/j.matchemphys.2018.11.045.

[38] R. Jain, N. Jadon, A. Pawaiya, Trends in Analytical Chemistry, 2017, 97, 363-373, doi: 10.1016/j.trac.2017.10.009.

[39] N. S. Seroka, M. A. Mamo, SN Applied Sciences, 2020, 2, 413, doi: 10.1007/s42452-020-2166-9.

[40] B. Li, K. Ma, S. Lu, X. Liu, Z. Ma, L. Zhang, X. Wang, S. Wang, Applied Physics A, 2020, 126, 791, doi: 10.1007/s00339020-03979-4.

[41] S. Seyedin, S. Uzun, A. Levitt, B. Anasori, G. Dion, Y. Gogotsi, J. M. Razal, Advanced Functional Materials, 2020, 30, 1910504, doi: 10.1002/adfm.201910504.

[42] S. Uzun, S. Seyedin, A. L. Stoltzfus, A. S. Levitt, M. Alhabeb, M. Anayee, C. J. Strobel, J. M. Razal, G. Dion, Y. Gogotsi, Advanced Functional Materials, 2019, 29, 1905015, doi: 10.1002/adfm.201905015.

[43] P. Sobolčiak, A. Tanvir, K. K. Sadasivuni, I. Krupa, Sensors, 2019, 19, 4589, doi: 10.3390/s19204589.

[44] Q. Li, R. Yin, D. Zhang, H. Liu, X. Chen, Y. Zheng, Z. Guo, C. Liu, C. Shen, Journal of Materials Chemistry A, 2020, 8, 21131-21141, doi: 10.1039/d0ta07832h.

[45] J. H. Pu, X. Zhao, X. J. Zha, L. Bai, K. Ke, R.-Y. Bao, Z. Y. Liu, M.-B. Yang, W. Yang, Journal of Materials Chemistry A, 2019, 7, 15913-15923, doi: 10.1039/c9ta04352g.

[46] T. Li, L. Chen, X. Yang, X. Chen, Z. Zhang, T. Zhao, X. Li, J. Zhang, Journal of Materials Chemistry C, 2019, 7, 1022-1027, doi: 10.1039/c8tc04893b.

[47] X. P. Li, Y. Li, X. Li, D. Song, P. Min, C. Hu, H. B. Zhang, N. Koratkar, Z. Z. Yu, Journal of Colloid and Interface Science, 2019, 542, 54-62, doi: 10.1016/j.jcis.2019.01.123.

[48] J. Yang, Y. Li, Y. Zheng, Y. Xu, Z. Zheng, X. Chen, W. Liu, Small, 2019, 15, 1902826, doi: 10.1002/smll.201902826.

[49] S. Zhao, H. B. Zhang, J. Q. Luo, Q. W. Wang, B. Xu, S. Hong, Z. Z. Yu, ACS Nano, 2018, 12, 11193-11202, doi: 10.1021/acsnano.8b05739.

[50] Y. Ma, Y. Yue, H. Zhang, F. Cheng, W. Zhao, J. Rao, S. Luo, J. Wang, X. Jiang, Z. Liu, N. Liu, Y. Gao, ACS Nano, 2018, 12, 3209-3216, doi: 10.1021/acsnano.7b06909.

[51] M. Hasanzadeh, N. Shadjou, M. de la Guardia, TrAC Trends in Analytical Chemistry, 2014, 62, 11-19, doi: 10.1016/j.trac.2014.06.011.

[52] Y. Zhang, K. Chen, Y. Li, J. Lan, B. Yan, L. Shi, R. Ran, ACS Applied Materials \& Interfaces, 2019, 11, 47350-47357, doi: 10.1021/acsami.9b16078.

[53] M. H. Tran, R. Brilmayer, L. Liu, H. Zhuang, C. Hess, A. Andrieu-Brunsen, C. S. Birkel, ACS Applied Nano Materials, 2020, 3, 4069-4076, doi: 10.1021/acsanm.0c00118. 
[54] Y. Ma, Y. Yue, H. Zhang, F. Cheng, W. Zhao, J. Rao, S. Luo, J. Wang, X. Jiang, Z. Liu, N. Liu, Y. Gao, ACS Nano, 2018, 12, 3209-3216, doi: 10.1021/acsnano.7b06909.

[55] K. Zhang, J. Sun, J. Song, C. Gao, Z. Wang, C. Song, Y. Wu, Y. Liu, ACS Applied Materials \& Interfaces, 2020, 12, 4530645314, doi: 10.1021/acsami.0c13653.

[56] L. Yuan, M. Zhang, T. Zhao, T. Li, H. Zhang, L. Chen, J. Zhang, Sensors and Actuators A: Physical, 2020, 315, 112192 , doi: 10.1016/j.sna.2020.112192.

[57] E. S. Muckley, M. Naguib, H.-W. Wang, L. Vlcek, N. C. Osti, R. L. Sacci, X. Sang, R. R. Unocic, Y. Xie, M. Tyagi, E. Mamontov, K. L. Page, P. R. C. Kent, J. Nanda, I. N. Ivanov, ACS Nano, 2017, 11, 11118-11126, doi: 10.1021/acsnano.7b05264.

[58] Y. Guo, M. Zhong, Z. Fang, P. Wan, G. Yu, Nano Letters, 2019, 19, 1143-1150, doi: 10.1021/acs.nanolett.8b04514.

[59] X. Shi, H. Wang, X. Xie, Q. Xue, J. Zhang, S. Kang, C. Wang, J. Liang, Y. Chen, ACS Nano, 2019, 13, 649-659, doi: 10.1021/acsnano.8b07805.

[60] Q. Wang, X. Pan, X. Wang, H. Gao, Y. Chen, L. Chen, Y. Ni, S. Cao, X. Ma, Composites Part B: Engineering, 2020, 197, 108187, doi: 10.1016/j.compositesb.2020.108187.

[61] N. Li, Y. Jiang, C. Zhou, Y. Xiao, B. Meng, Z. Wang, D. Huang, C. Xing, Z. Peng, ACS Applied Materials \& Interfaces, 2019, 11, 38116-38125, doi: 10.1021/acsami.9b12168.

[62] A. P. Nayak, Z. Yuan, B. Cao, J. Liu, J. Wu, S. T. Moran, T. Li, D. Akinwande, C. Jin, J. F. Lin, ACS Nano, 2015, 9, $9117-$ 9123, doi: 10.1021/acsnano.5b03295.

[63] J. Yan, Y. Ma, X. Li, C. Zhang, M. Cao, W. Chen, S. Luo, M. Zhu, Y. Gao, Ceramics International, 2020, 46, 23592-23598, doi: 10.1016/j.ceramint.2020.06.131.

[64] D. Song, X. Li, X. P. Li, X. Jia, P. Min, Journal of Colloid and Interface Science, 2019, 555, 751-758, doi: 10.1016/j.jcis.2019.08.020.

[65] S. Verma, S. Sinha-Ray, S. Sinha-Ray, Polymers, 2020, 12, 238, doi: 10.3390/polym12010238.

[66] S. Chen, R. Li, X. Li, J. Xie, Advanced Drug Delivery Reviews, 2018, 132, 188-213, doi: 10.1016/j.addr.2018.05.001.

[67] J.-W. Jung, C.-L. Lee, S. Yu, I.-D. Kim, Journal of Materials Chemistry A, 2016, 4, 703-750, doi: 10.1039/c5ta06844d.

[68] K. Yang, F. Yin, D. Xia, H. Peng, J. Yang, W. Yuan, Nanoscale, 2019, 11, 9949-9957, doi: 10.1039/c9nr00488b.

[69] S. Wang, X. Du, Y. Luo, S. Lin, M. Zhou, Z. Du, X. Cheng, H. Wang, Chemical Engineering Journal, 2021, 408, 127363, doi: 10.1016/j.cej.2020.127363.

[70] R. Liu, J. Li, M. Li, Q. Zhang, G. Shi, Y. Li, C. Hou, H. Wang, ACS Applied Materials \& Interfaces, 2020, 12, 46446-46454, doi: 10.1021/acsami.0c11715.

[71] S. Sharma, A. Chhetry, M. Sharifuzzaman, H. Yoon, J. Y. Park, ACS Applied Materials \& Interfaces, 2020, 12, $22212-$ 22224, doi: 10.1021/acsami.0c05819.

[72] X. Zheng, J. Shen, Q. Hu, W. Nie, Z. Wang, L. Zou, C. Li, Nanoscale, 2021, 13, 1832-1841, doi: 10.1039/d0nr07433k.

[73]H Li, Z. Du, ACS Applied Materials \& Interfaces, 2019, 11, 45930-45938, doi: 10.1021/acsami.9b19242.
[74] R. B. Rakhi, P. Nayak, C. Xia, H. N. Alshareef, Scientific Reports, 2016, 6, 36422, doi: 10.1038/srep36422.

[75] M. Li, L. Fang, H. Zhou, F. Wu, Y. Lu, H. Luo, Y. Zhang, B. Hu, Applied Surface Science, 2019, 495, 143554, doi: 10.1016/j.apsusc.2019.143554.

[76] S. Kadirsoy, N. Atar, M. L. Yola, New Journal of Chemistry, 2020, 44, 6524-6532, doi: 10.1039/d0nj00951b.

[77] X. Ma, X. Tu, F. Gao, Y. Xie, X. Huang, C. Fernandez, F. Qu, G. Liu, L. Lu, Y. Yu, Sensors and Actuators B: Chemical, 2020, 309, 127815, doi: 10.1016/j.snb.2020.127815.

[78] A. K. Sharma, B. Kaur, C. Marques, Optik, 2020, 218, 164891, doi: 10.1016/j.ijleo.2020.164891.

[79] M. Wu, M. He, Q. Hu, Q. Wu, G. Sun, L. Xie, Z. Zhang, Z. Zhu, A. Zhou, ACS Sensors, 2019, 4, 2763-2770, doi: 10.1021/acssensors.9b01308.

[80] B. Xiao, Y.-C. Li, X.-F. Yu, J.-B. Cheng, Sensors and Actuators B: Chemical, 2016, 235, 103-109, doi: 10.1016/j.snb.2016.05.062.

[81] X. Chen, X. Sun, W. Xu, G. Pan, D. Zhou, J. Zhu, H. Wang, X. Bai, B. Dong, H. Song, Nanoscale, 2018, 10, 1111-1118, doi: 10.1039/c7nr06958h.

[82] W. Yuan, K. Yang, H. Peng, F. Li, F. Yin, Journal of Materials Chemistry A, 2018, 6, 18116-18124, doi: 10.1039/c8ta06928j.

[83] Y. Fang, X. Yang, T. Chen, G. Xu, M. Liu, J. Liu, Y. Xu, Sensors and Actuators B: Chemical, 2018, 263, 400-407, doi: 10.1016/j.snb.2018.02.102.

[84] B. D. Sahu, A. K. Kalvala, M. Koneru, J. Mahesh Kumar, M. Kuncha, S. S. Rachamalla, R. Sistla, PLoS One, 2014, 9, e105070, doi: 10.1371/journal.pone.0105070.

[85] E. Lee, A. VahidMohammadi, D. Lee, J. Yoon, M. Beidaghi, Y. S. Yoon, D. J. Kim, ECS Meeting Abstracts, 2019, MA201902, 2225, doi: 10.1149/ma2019-02/51/2225.

\section{Author information}

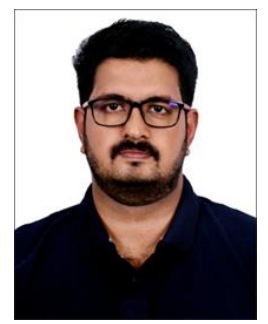

Vishnu Sankar Sivasankarapillai received his B.Sc and M.Sc Chemistry degrees from Mahathma Gandhi university, Kerala, India. He is currently working towards Ph.D. degree under the supervision of Dr. Ragupathy Dhanusuraman, Department of Chemistry at National Institute of Technology Puducherry, India. His research interests include Biomaterials and Nanocomposites for energy and biomedical applications.

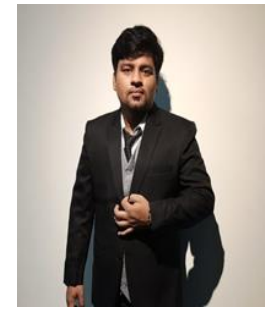

Tata Sanjay Kanna Sharma received his B.S. (2012), and M.S. (2014), degrees from Sri Venkateswara University, Tirupathi, India and acquired Doctor of Philosophy Ph.D. (2021) from National Taipei University of Technology, Taipei, Taiwan. His current research Interest mainly focuses on the synthesis of transition metal/Rare earth 
vanadate-carbon based Aerogels, Novel metal oxides combined with green synthesized carbon sources for real-time analysis in biosensors. He is more interested in Optical properties of Graphene, Landau levels for sensor applications and drug delivery.

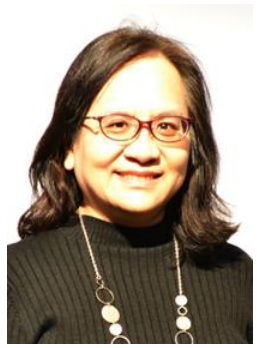

Hwa-Yuan Kuo is an Associate Professor and the director of the Center for Biomedical Industries at the National Taipei University ofTechnology. Dr. $H$ wagraduated and received her PhD from the School of Medicine, the Johns Hopkins University. She is the president of theMedical Association for Indigenous Peoples of Taiwan (MAIPT). Dr. Hwa's scientific interests are: 1) nanotechnology and biosensor, 2) newdrug discovery for human diseases by proteomics and genomics approaches and 3) glycobiology, especially on enzymes kinetics.She has published $100+$ conference and journal articles and 15 patents.

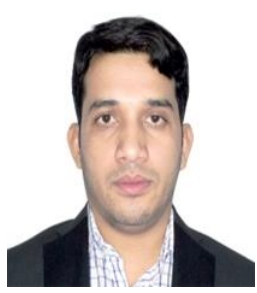

Saikh Mohammad Wabaidur is working as Researcher at King Saud University, Saudi Arabia. He received his PhD in 2021, from Banasthali University, India. He has published 110 research articles in international journals. His research field includes Separation, identification and determination of biologically important compounds and pharmaceutical drugs.

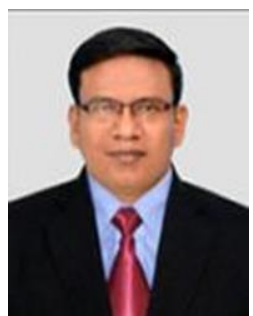

Subramania Angaiah has been working in the field of Materials Electrochemistry for the last 20 years. His area of research includes M-ion batteries, Supercapacitors, Dye-sensitized solar cells, Nanocomposites, Electrospun polymer membrane electrolytes for electrochemical energy devices, Corrosion and Surface Engineering etc. He has published more than 130 research articles in the peerreviewed international journals.

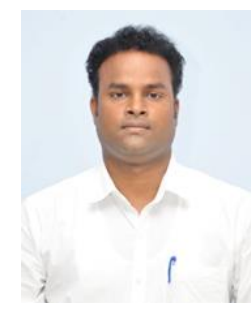

Ragupathy Dhanusuraman is Assistant professor and Head at the Department of Chemistry, National Institute of Technology Puducherry, Karaikal, India. He received his PhD in 2010, from Kyungpook National University, South Korea. He has published 75 research articles in international journals. His research field includes the development of newer nanomaterials for energy, biosensor and electro-catalytic applications.
Publisher's Note: Engineered Science Publisher remains neutral with regard to jurisdictional claims in published maps and institutional affiliations. 